\title{
Optical Characteristics of InGaN/GaN Quantum Well Structures with Embedded Quantum Dots
}

\author{
Yi-Yin Chung ${ }^{1}$, Yen-Sheng Lin $^{2}$, Shih-Wei Feng ${ }^{1}$, Yung-Chen Cheng ${ }^{1}$, Kung-Jen $\mathrm{Ma}^{3}$, C. C. Yang ${ }^{1}$, \\ Cheng-Ta Kuo ${ }^{4}$ and Jian-Shihn Tsang ${ }^{4}$ \\ ${ }^{1}$ Graduate Institute of Electro-Optical Engineering and Department of Electrical Engineering, \\ National Taiwan University, 1, Roosevelt Road, Sec. 4, Taipei, Taiwan \\ (phone) 886-2-23657624 (fax) 886-2-23652637 (E-mail) ccy@cc.ee.ntu.edu.tw \\ ${ }^{2}$ Department of Electrical Engineering, Chinese Naval Academy (Taiwan), Kaohsiung, Taiwan \\ ${ }^{3}$ Department of Mechanical Engineering, Chung Hua University, Hsinchu, Taiwan \\ ${ }^{4}$ Advanced Epitaxy Technology Inc., Hsinchu Industrial Park, Taiwan
}

\begin{abstract}
We compared the results of optical characterization between five InGaN/GaN quantum well samples of different well widths. Temperature dependencies of photoluminescence (PL) spectral positions, integrated PL intensities, and PL intensity decay times at PL peaks of all the five samples showed three temperature ranges of different variation trends. The radiative efficiencies of the samples in the high temperature range had the same decay slope, which is supposed to be determined by the defect structures outside clusters. The radiative efficiencies in the medium temperature range varied among samples, indicating different defect structures in the regions between coupled clusters in different samples. Consistent results of temperature dependent variations between the integrated PL intensity and PL decay time among these samples provided clues for reasonable interpretations. Also, we showed the strong dependencies of thermal annealing effects on quantum well (QW) width in InGaN/GaN QW structures. Thermal annealing at $800{ }^{\circ} \mathrm{C}$ of a narrow $\mathrm{QW}$ width $(2 \mathrm{~nm})$ structure led to improved optical quality. However, thermal annealing at the same temperature of a sample of larger QW width (4 $\mathrm{nm}$ ) resulted in degraded optical quality.
\end{abstract}

Keywords: InGaN/GaN quantum well, indium aggregation cluster, quantum dot, decay time, thermal annealing

\section{INTRODUCTION}

Photon emission characteristics of InGaN/GaN quantum wells (QWs) have been widely studied because of the important applications of such a compound structure to light emitting devices. Due to the large lattice constant difference and hence low miscibility between $\mathrm{GaN}$ and $\mathrm{InN}$, indium aggregations around $\mathrm{InGaN} \mathrm{QW}$ layers, which result in carrier localization $(C L)$, have been widely observed [1-4]. Also, the strain-induced piezoelectric field, which generates the quantum confined Stark effect (QCSE), has been used for interpreting measured optical phenomena [5,6]. It is expected that both effects of CL and QCSE play certain roles in photon emission mechanisms of such a compound structure. In this regard, the dependence of optical properties on QW width has been an issue of focus in the related research. In this paper, we report systematical results of optical analyses of five InGaN/GaN QW structures of the same indium content but different QW widths and point out several novel discoveries. Consistent results from the measurements of photoluminescence (PL) will be discussed. We also report the different thermal annealing results in InGaN/GaN QW structures of different QW widths. Because of different levels of built strain energy in different samples, thermal annealing resulted in different cluster structures and hence quite different optical properties. Continuous PL were conducted to show various optical behaviors.

\section{EXPERIMENTAL RESULTS - QW WIDTH DEPENDENCE}

The samples were grown on c-plane sapphire with metal organic chemical vapor deposition. Following the deposition of $30 \mathrm{~nm} \mathrm{GaN}$ buffer layer and a $2.3 \mu \mathrm{m}$ GaN layer, QW structures consisting of five pairs of $\mathrm{In}_{0.15} \mathrm{Ga}_{0.85} \mathrm{~N}$ well and $10 \mathrm{~nm}$-thick $\mathrm{GaN}$ barrier were grown. Five samples of different well widths at 2, 2.5, 3, 3.5, and $4 \mathrm{~nm}$ were prepared and referred to as samples w20, w25, w30, w35, and w40, respectively. Fig. 1 shows the temperature-dependent PL peak positions of these samples. The PL peaks of all samples show S-shape behaviors. The S-shape temperature dependence has been interpreted by both mechanisms of CL and QCSE. The PL peak of w40 keeps blue shift in the temperature range from 80 up to $300 \mathrm{~K}$, implying that localized carriers may need more thermal energy to overcome the potential barriers in this sample. In other words, indium-rich clusters in sample w40 may provide stronger carrier confinement than other samples. Meanwhile, such a 
blue-shift behavior can be attributed to the strong QCSE in this sample. Thermal relaxation of QCSE in this sample lasts for a large temperature range.

Fig. 2 shows normalized integrated PL intensity (internal quantum efficiency) variations with temperature. In Fig. 2, the integrated PL intensities show three-step decay features. The three steps are divided by the temperatures at $\mathrm{T}_{1}=80 \mathrm{~K}$ and $\mathrm{T}_{2}$ $=180 \mathrm{~K}$. These two temperatures also roughly divide the PL peak variations of all samples (except w40) in Fig. 1 into three ranges: red shift-blue shift-red shift. The integrated PL intensity decay profiles of all samples in the low temperature range $\left(\mathrm{T}<\mathrm{T}_{1}\right)$ are almost the same. Those in the high temperature range $\left(\mathrm{T}>\mathrm{T}_{2}\right)$ are parallel (in the $\log$ scale). In the medium temperature range, transition decay slopes among different samples can be seen. The decay of w25 in the medium temperature range basically extends from the low temperature range. For w30, the same decay slope covers the medium and high temperature ranges. For the other three samples, the slopes in the medium temperature range are about the same and are steeper than that in the high temperature range. Sample w20 shows a transition temperature range from 130 through $210 \mathrm{~K}$ for changing the decay slope. It is interesting to note that the almost linear dependencies of normalized integrated PL intensities in Fig. 2 mean that the widely used non-radiative model of activation energy is not applicable in fitting our data. The different behaviors between the medium and high temperature ranges may imply that there are two different types of defects in the samples. The defects controlling the behaviors in the medium temperature range are located within clusters and between the coupled clusters. Those controlling the behaviors in the high temperature range are located outside clusters. The comparisons among different samples suggest that there be different densities of defects.

\section{EXPERIMENTAL RESULTS - THERMALLY ANNEALING EFFECTS}

As-grown samples were thermally annealed in a quartz tube furnace at different temperatures ranging from 800 to $900{ }^{\circ} \mathrm{C}$ in nitrogen ambient for $30 \mathrm{~min}$. PL spectral peak variations with temperature of the three samples under various thermal annealing conditions are shown in Fig. 3. After thermal annealing, the S-shape variation of PL peak is maintained in each case. Thermal annealing results in blue shifts of PL peak in w20 and red shifts in w30 and w40. Also, in all samples thermal annealing at $800{ }^{\circ} \mathrm{C}$ leads to the largest spectral shifts. As thermal annealing temperature increases, the blue shift or red shift is reduced. Fig. 4 shows the temperature-dependent variations of integrated PL intensity of the as-grown and annealed samples. The integrated PL intensities were enhanced upon thermal annealing in w20 and w30, particularly significant in w20. However, that of w40 was reduced. From Figs. 3 and 4, it is interesting to note that the trends of PL peak shifts (blue or red) and integrated PL intensity variations (increase or decrease) with annealing temperature are the same in each of the three samples. Thermal annealing at $800{ }^{\circ} \mathrm{C}$ always leads to the largest changes. As annealing temperature increases, optical properties generally tend to return to the as-grown condition.

In w20, upon thermal annealing, spinodal decomposition may dominate the process of relaxing the strain energy built in the well layers. In this process, the "up-hill" diffusion results in the gathering of indium toward their cores and forms the more regular structures. However, in w40 because the larger well width leads to larger strain energy, spinodal decomposition becomes less effective. In this situation, the strain energy might be relaxed through the formation of more stacking faults or local lattice distortions upon thermal annealing. Stacking faults and local distortions were observed in atomic-scale HRTEM images (not shown) of as-grown and annealed w40 samples. It is speculated that the thermally annealed w40 may have a higher density of such defects. Such defects exist around QDs and are responsible for the significant integrated PL intensity decrease with temperature near $50 \mathrm{~K}$, as shown in Fig. 4. Note that in the aforementioned relaxation process of w40, the local strain distribution around a QD may be enhanced although the global stress is reduced. Therefore, the reduction of integrated PL intensity of w40 upon thermal annealing can be attributed to the increase of either local strain (reduced radiative transition rate due to the enhanced QCSE) or defect density.

The blue shift of PL peak in w20 upon thermal annealing can be attributed to the better quantum confinement. The causes of red shifts in w30 and w40 could be quite complicated. They result from the interplay of indium content gathering (red shift trend), quantum confinement (blue shift trend) and strain-induced QCSE (red shift trend). Based on the microstructure observations, the enhanced QCSE may play the key role here. The increasing trends of integrated PL intensities and PL decay times of w20 upon thermal annealing are consistent. These trends may originate from the mixed effects of the variations of CL and QCSE after thermal annealing. Stronger CL further prevents carriers from non-radiative recombination and hence stronger PL intensities and longer decay times. A weaker QCSE increases radiative transition rate and hence stronger PL intensity. However, it reduces the PL decay times. Because the PL decay times were increased upon thermal annealing, the effect of enhanced CL seems to play the key role in annealed w20 samples. This conclusion is consistent with the well-shaped QDs in annealed w20. It is also consistent with a previous conclusion that CL dominates in optical properties in a thin well sample. Note that in w30 because the red shifts of PL peak are quite large and the increases of integrated PL intensity are relatively smaller upon thermal annealing, the enhanced local strain-induced QCSE may dominate over $C L$ in the annealing effects of this sample. 


\section{CONCLUSIONS}

In summary, we have compared the results of optical and material characterization between five InGaN/GaN QW samples of the same indium content but different well widths. Material analyses showed that narrower and sharper clusters were formed in wider well samples, which resulted in stronger CL and QCSE and hence longer PL decay times. The PL intensity variations with $T$ indicate that defects around clusters play an important role in optical properties. Also, we have shown the dependencies of optical and material properties of thermally annealed InGaN/GaN QW samples on well width. In each sample of different QW widths, the results of temperature variations of PL spectral peak, integrated PL intensity and PL decay time showed consistent trends in varying annealing temperature. Different variation trends upon thermal annealing in different samples were also consistent with the results of HRTEM images. Generally speaking, thermal annealing at $800{ }^{\circ} \mathrm{C}$ of a narrow QW width $(2 \mathrm{~nm}$ ) structure led to regularly distributed QDs and improved optical quality. On the other hand, thermal annealing at $800{ }^{\circ} \mathrm{C}$ of a sample of larger QW width $(4 \mathrm{~nm})$ resulted in QD structures of even higher local strains and degraded optical quality.

\section{ACKNOWLEDGEMENTS}

This research was supported by National Science Council, The Republic of China, under the grants of NSC 89-2218-E-002-094, NSC 89-2218-E-002-095, and NSC 89-2215-E-002-051.

\section{REFERENCES}

1. Y. Narukawa, Y. Kawakami, M. Funato, S. Fujita, S. Fujita, and S. Nakamura, Appl. Phys. Lett. 70, 981 (1997).

2. Y. S. Lin, K. J. Ma, C. Hsu, S. W. Feng, Y. C. Cheng, C. C. Liao, C. C. Yang, C. C. Chou, C. M. Lee, and J. I. Chyi, Appl. Phys. Lett. 77, 2988 (2000).

3. Y. Narukawa, Y. Kawakami, S. Fujita, S. Fujita and S. Nakamura, Phys. Rev. B 55, R1938 (1997).

4. T. Hino, S. Tomiya, T. Miyajima, K. Yanashima, S. Hashimoto, and M. Ikeda, Appl. Phys. Lett. 76, 3421 (2000).

5. S. F. Chichibu, A. C. Abare, M. S. Minsky, S. Keller, S. B. Fleischer, J. E. Bowers, E. Hu, U. K. Mishra, L. A. Coldren and S. P. DenBaars, Appl. Phys. Lett. 73, 2006 (1998).

6. P. Riblet, H. Hirayama, A. Kinoshita, A. Hirata, T. Sugano, and Y. Aoyagi, Appl. Phys. Lett. 75, 2241 (1999).

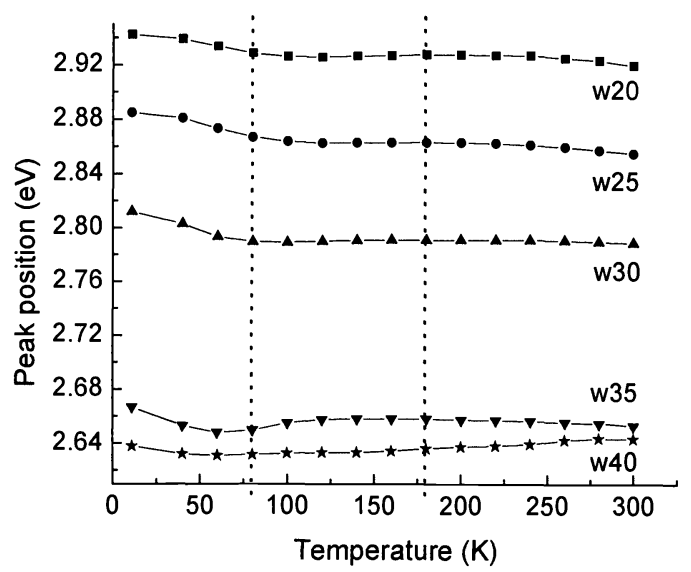

Fig. 1 Variations of PL spectral peak versus temperature of the five samples.

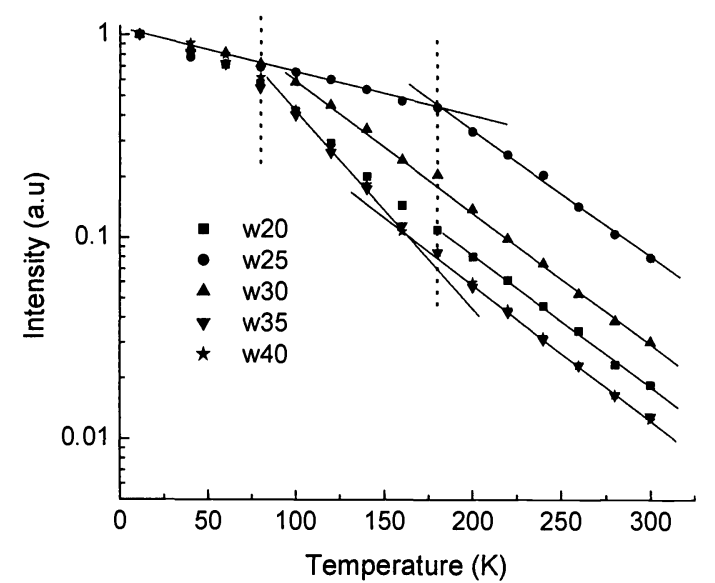

Fig. 2 Variations of normalized integrated PL intensity versus temperature of the five samples. Straight lines are drawn to guide eyes. 


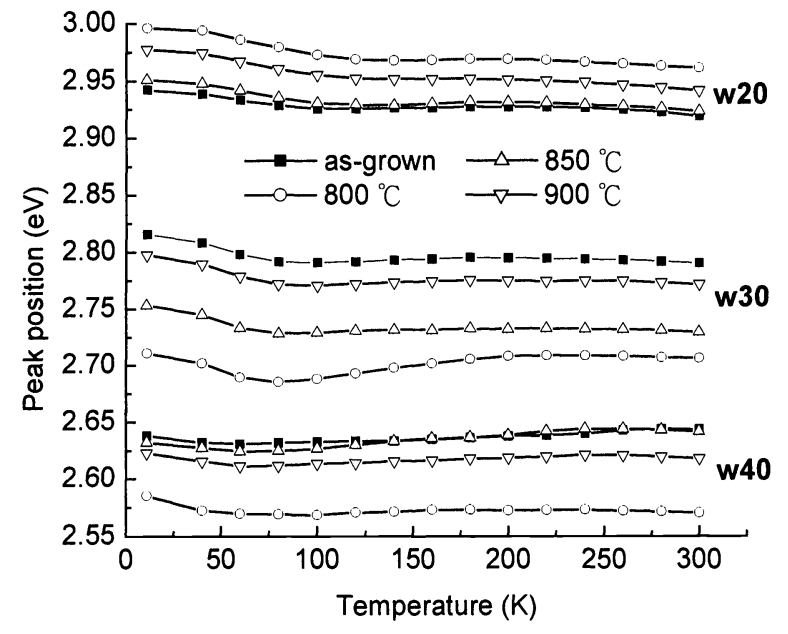

Fig. 3 Variations of PL peak positions versus temperature of the three samples under various thermally annealing conditions.

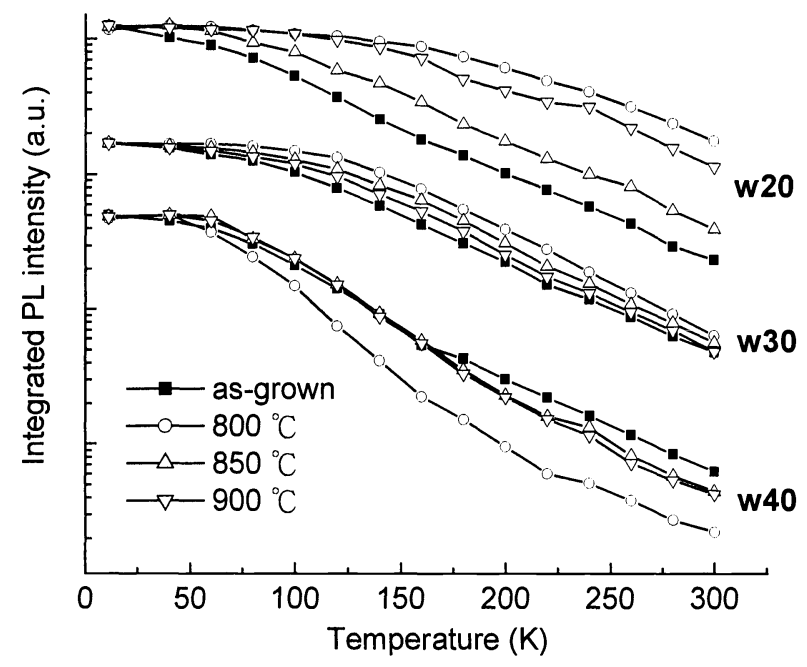

Fig. 4 Variations of normalized integrated PL intensities versus temperature of the three samples under various thermally annealing conditions. 